\title{
Article
}

\section{A method of creating personae for children with autism spectrum condition: application and revision}

Zubair, Misbahu Sharfuddeen, Brown, David, Hughes-Roberts, Thomas and Bates, Matthew

Available at http://clok.uclan.ac.uk/32918/

Zubair, Misbahu Sharfuddeen ORCID: 0000-0001-7604-0592, Brown, David, Hughes-Roberts, Thomas and Bates, Matthew (2019) A method of creating personae for children with autism spectrum condition: application and revision. Journal of Enabling Technologies, 13 (2). pp. 112-122. ISSN 23986263

It is advisable to refer to the publisher's version if you intend to cite from the work. http://dx.doi.org/10.1108/JET-12-2018-0062

For more information about UCLan's research in this area go to http://www.uclan.ac.uk/researchgroups/ and search for <name of research Group>.

For information about Research generally at UCLan please go to http://www.uclan.ac.uk/research/

All outputs in CLoK are protected by Intellectual Property Rights law, including Copyright law. Copyright, IPR and Moral Rights for the works on this site are retained by the individual authors and/or other copyright owners. Terms and conditions for use of this material are defined in the policies page.

\section{CLoK}

Central Lancashire online Knowledge www.clok.uclan.ac.uk

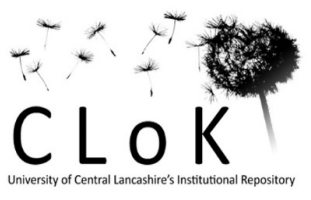


A method of creating personae for children with autism spectrum condition: application and revision

\begin{tabular}{|r|l|}
\hline Journal: & Journal of Enabling Technologies \\
\hline Manuscript ID & JET-12-2018-0062.R1 \\
\hline Manuscript Type: & Peer-Reviewed Paper \\
\hline Keywords: & $\begin{array}{l}\text { Persona, Autism, Visual Programming, User modeling, Accessibility, } \\
\text { Usability }\end{array}$ \\
\hline \multicolumn{2}{|l}{} \\
\hline
\end{tabular}

SCHOLARONE ${ }^{\text {m }}$

Manuscripts 


\title{
A method of creating personae for children with autism spectrum condition: application and revision
}

\begin{abstract}
Purpose: Personae are simple tools for describing users, their characteristics and their goals. They are valuable tools when designing for a specific group of users, such as children with autism spectrum condition. The purpose of this paper is to propose, validate and revise a methodology for creating accurate, data grounded personae for children with autism spectrum condition.
\end{abstract}

Methodology: The proposed method is based mainly on Cooper et al.'s (2007) persona construction method. It proposes gathering and analysing qualitative data from users and experts to either create a new persona or extend an existing one. The method is then applied to create personae for the design of a visual programming tool for children with autism spectrum condition. Based on the results of the application, observations, and lessons learnt, a revised version of the method is proposed.

Findings: The method's combined use of user data and expert knowledge produced a set of personae that have been well reviewed by experts so far. The method's use of a questionnaire to validate personae also produced relevant qualitative feedback. On review, possible downsides of extending existing personae were identified. Therefore, a revised method was introduced, eliminating the need to extend existing personae, and stressing the importance of utilising user data, expert knowledge and feedback.

Value: This paper addresses the need for a well-defined method for creating data grounded personae that accurately describe the characteristics and goals of children with autism spectrum condition. Such personae can be used to design and develop more accessible and usable products.

\section{Introduction}

Cooper (1999) introduced the role of personae in his book The Inmates are Running the Asylum. He described personae as simple tools that provide a "precise description of our user and what he wishes to accomplish" (Cooper, 1999). Personae paint a "memorable, engaging and actionable image" of the user being designed for (Adlin and Pruitt, 2010). Each persona describes a hypothetical archetype of real users (Cooper, 1999) i.e. it represents a group of users that share similar behaviours towards the use of a particular product or service (Cooper et al., 2007). Unlike other models of user representation such as profiles which are made up of lists of attributes, a persona narrates a realistic and relatable description of an individual user. It includes a name, a background story, characteristics, needs and goals of the user related to the product or service being designed, and in some cases a photographic image of the user. This personification serves as a way to vividly relay relevant information about the persona as well as a way of drawing empathy, and interest from designers (Adlin and Pruitt, 2010; Cooper et al., 2007).

\subsection{Applications of Personae}

Personae have numerous application potentials in user centred design. Personae can be used to explicitly define and set focus on a specific set of users and their goals (Adlin and Pruitt, 2010; Cooper et al., 2007). Without such a clear focus on target users, there is the risk of designing a product for "the elastic user" (Cooper, 1999). The elastic user is an imaginary target user whose 
needs and capabilities are reimagined at the convenience of the designer. One moment the elastic user is a beginner who requires guiding instructions at every step of the way, at another moment the elastic user becomes an expert able to find his way blindly through the maze of a configurations page.

Using personae is a great way of having designers think about their target users at a personal level. Referring to their personae as individuals, they are able to make user centred decisions by asking and answering questions such as "Will Sue use this feature?" or "Is this going to be too complicated for Frank?" (Floyd et al., 2008). Therefore personae can lead to better design decisions, and help avoid decisions based on assumptions about users (Adlin and Pruitt, 2010).

Personae are not useful for designers alone, they can be used by developers, product managers, executives, and all other stakeholders, especially as tools for communicating about users (Cooper, 1999). Communication can be easier and more effective if everyone involved is familiar with the personae being designed for, and more importantly, treats each persona as a real individual. Personae can also ensure all stakeholders are in agreement regarding features needed and not needed to satisfy user needs. Certain features might have huge priorities assigned to them by certain stakeholders, although they might not be of high priority to the target user. Personae can help all stakeholders step into the user's shoes and reassess feature choices and priorities. To do so, questions can be asked of features, for example, "Does Sam need to be able to write his report in 20 languages?" or "Is Jill going to prefer voice or gesture control?". This can be a way of ending feature debates amongst stakeholders (Cooper, 1999).

\subsection{Using Personae to Design for Children with Autism Spectrum Conditions}

Since personae help put target users at the centre of the design process, they can be valuable tools when designing for individuals with special needs, such as children with autism spectrum condition (ASC). ASC is a condition that is characterised by deficits in "social communication and social interaction" and "restricted, repetitive patterns of behaviour, interests, or activities" (American Psychiatric Association, 2014). For each child with ASC, these characteristics can be unique in the way they manifest and in their severity. Children with ASC may also have accompanying intellectual disability (American Psychiatric Association, 2014). This means that children with ASC are a diverse group of individuals with varying needs and capabilities. For the designer, this can be difficult to empathise with if given a list of characteristics and needs. The designer may focus too much on the list of attributes and in the process ignore the user as a unique individual.

To design accessible and usable products for children with ASC, personae can provide lifelike design targets for designers. For example, McCrickard et al. (2015) reported the use of persona in educating novice user-interface designers about their target users while designing an anger management solution for children with ASC. In another case, Vieira et al. (2017) proposed a multimodal interactive application for children with ASC by designing for users represented by Nuno; a persona created by Leal et al. (2016) describing a 10-year-old child with ASC.

\subsection{Personae Creation Methods and Implications}

Personae are imaginary representations of users, but Cooper (1999) argues that they should be built through a methodical approach of analysing data gathered from real users. However, not all personae are built from real user data, and the method used to build personae varies. One major determinant of the data and method used to build a persona is the intended use of the persona (Floyd et al., 2008). 
For instance, Norman's ad-hoc persona is used to make designer's assumptions and intuitions explicit, aid communication and define possible use cases (Norman, 2006). It is not meant to represent real users and is not used as such. Therefore it is not built on real user data but rather built using the designer's imagination, intuition, experience and/or stereotypical behaviours of the target user group. The extreme persona by Djajadiningrat et al. (2004) is also not aimed at representing real users, it is used to describe extreme characters and test behavioural boundaries. Hence it is made up of mainly imaginary data.

However, in situations where personae are used as real user representatives for the purposes of identifying user requirements, the personae must be built using real user data. Cooper et al.'s (2007) goal-based persona is meant specifically for this purpose. It is built on real user data gathered through ethnographic interviews in order to ensure that it accurately represents the characteristics and goals of the target user. The gathered data is analysed to identify significant user behaviours and groups of users with similar behaviours. A persona is then created for each group of similar users, i.e. having the main behavioural characteristics, needs and goals common to the group. Fictional personal information and background story are added to personify each created persona.

The role-based persona by Adlin and Pruitt (2010) also serve as a real user representative. Unlike the goal-based persona (Cooper et al., 2007), the role-based persona uses both qualitative and quantitative sources of user data. Categories and sub-categories of users are created based on similarities of users and their needs extracted from analysing the data. Persona skeletons, which contain brief lists of attributes or assumptions about sets of users within each sub-category are then created, prioritised, and developed into full personae. Lastly, created personae are validated against real user data or by experts on the target users.

Although personae have been used as real user representatives when designing for children with ASC (Al-Wabil et al., 2012; McCrickard et al., 2015; Vieira et al., 2017), there is still little information on the methods used for creating accurate data grounded personae for children with ASC. Therefore there is the need for a novel method for creating accurate data grounded personae for children with ASC. It should take into account the difficulties, capabilities and needs of this target group in its procedures (e.g. data collection and analysis) and in its end product.

The aim of this paper is to present the following: a proposed method for creating personae for children with ASC, validation of the method, and a revised version of the method. The remainder of this paper contains the proposed method of personae creation, a summary of the method's application and emerging results, followed by an analysis of the lessons learnt, observations and reflections from the method's application. A revised version of the method is then presented before the paper is concluded.

\section{Proposed Method}

The method proposed in this work is intended for building data grounded personae for children with ASC. It takes from Cooper et al.'s (2007) steps for constructing personae from empirical data, and Adlin and Pruitt's (2010) subject matter expert (referred to as experts from here on) approach to validation. The method uses an improved version of Leal et al.'s (2016) method of expert validation. It also recommends the involvement of experts in various stages of the construction process in addition to the validation stage. Lastly, the method provides the possibility of reusing existing personae. 
The proposed method comprises a set of tasks performed on a set of gathered data (see Figure 1). Both tasks and required data are briefly discussed below:

Figure 1 showing the proposed method based on Cooper et al.'s (2007) method of constructing personae.

\subsection{Data Requirements}

The importance of using data collected from real target users for constructing personae cannot be overstated, and the best methods for collecting such data are interviews and observations. However, when it comes to children with cognitive impairments of any kind, interviews are not likely to produce a meaningful qualitative data set. Therefore, this method recommends collecting data from target users through observations.

Additional data should then be collected from experts. Data from experts can play three major roles: add to what is known about the target users, clarify data collected through user observations, and validate the accuracy of constructed personae. Interviews or focus groups should be used to collect data from experts aimed at adding knowledge or clarifying user data. While any (or a combination) of interviews focus groups, surveys and questionnaires can be used to collect supplementary data for validating personae.

Existing personae are also part of the data requirements of this method. They can be gathered through an extensive literature search.

\subsection{Tasks}

The method has eight (8) sequential tasks based on the tasks for constructing personae proposed by Cooper et al. (2007). At least seven of the eight tasks must be performed to construct a persona. Either Task 6 or Task 7 is bypassed depending on the outcome of Task 5. The tasks are briefly discussed below:

Task 1 - Identify and list significant user behaviours from observational data: Analyse the gathered observational user data with the aim of identifying and listing unique significant behaviours exhibited by users. The aim is not to list all behaviours, but only those that are significant to the product or service being designed. The resulting list becomes the list of "behavioural variables" (Cooper et al., 2007) and will be used in some of the subsequent tasks. Cooper et al. (2007) proposed types of behavioural variables that can be used to distinguish behaviours, they are activities, attitudes, aptitudes, motivations and skill.

For example, consider analysing observational data of users using a task management application. Behaviours that fall under the "Activities" type can be things like creating a task, sharing task details, creating a reminder, checking free time etc. Behaviours that fall under "Motivations" can be things like planning the day, tracking school timetable, tracking house chores etc. It should be noted however that these categories may not be perfect for all users, products, or context of use. Therefore, behavioural variables types may need to be added accordingly.

Task 2 - Map observed users to the identified behavioural variables: Show the behaviours exhibited by each observed user by mapping users to the identified behavioural variables in Task 1. Mappings should be informed purely by the data collected on each user. The aim of this step is to show the behaviour of each user in relation to other users i.e. whether user $A$ exhibits a behaviour more, less or about the same amount as users B, C, D. This allows users with similar behaviours to be spotted easily.

For example, in the task management app scenario, you may have the following users: John who uses the app as a school requirement creates tasks related to school activities, and marks them as 
completed when they are done; Alexa who uses the app to plan her day, creates tasks regularly, uses reminders, shares task details with her family and best friend; and another user Jane who receives tasks from her mum, marks them as done when completed, shares the status of her tasks with her mum, and checks her available free time.

Task 3 - Identify and group users with similar meaningful patterns of behaviour: Using the user to behaviour mappings created in Task 2, identify users with similar meaningful-patterns of behaviour. Patterns of behaviour with no reasonable explanation should be avoided. For example, in the task management app scenario, users who create and update their own tasks can be grouped with John and Alexa, while users who receive tasks and send task status updates can be grouped with Jane.

The example above is a straightforward one, however, in some cases differentiating between meaningful and non-meaningful patterns of behaviour when dealing with children with ASC may not be as straight forward. Therefore we recommend taking advantage of the experience and background knowledge of experts in identifying meaningful patterns that may not be obvious to non-experts.

Task 4 - List the characteristics and goals of the identified groups to form persona abstracts: For each group of users identified in Task 3, create a persona abstract by listing the characteristics and goals associated with each of its patterns of behaviour. Characteristics and goals are usually the reasons behind the group's behaviours and can be extracted from the user data. However, since the user data being analysed is observational data, extracting user characteristics and goals may not be as straight forward as extracting from data gathered through interviews, where users can explicitly provide the needed information. Therefore, the knowledge and experience of experts are needed in this task to analyse the information known about users and inform on the characteristics and goals leading to such behaviour.

Once characteristics and goals have been listed, personify the persona abstracts by providing each with basic personal information (fictional) such as name and age, and some background details.

Task 5 - Compare persona abstracts with existing personae: Compare each persona abstract's listed characteristics and goals with those described in the personae from the set of gathered existing personae. Existing personae should be for the same target users and similar context of use. The aim of this task is to identify personae that closely match the persona abstracts. Personal information, which is fictional should not be used to determine similarities or lack of. The focus should be strictly on comparing characteristics and goals.

Task 6 - Extend existing personae to fully describe the persona abstracts: If an existing persona is found that is similar to a persona abstract, modify the existing persona's descriptions, characteristics and goals so that it now fully describes the characteristics and goals of the persona abstract. For an existing persona to be chosen for this task it needs to be very similar in characteristics and goals with the persona abstract.

Task 7 - Fully describe the characteristics and goals of persona abstracts: For each persona abstract without a similar counterpart in the set of existing personae, construct rich descriptions for its listed characteristics and goals to form a full persona. Descriptions should be in the form of a third person narrative that uses fictional situations in explaining the characteristics and goals of the persona. Also, provide a fictional background story to make the persona more lifelike. 
Task 8 - Validate personae by getting expert opinion and feedback: Finally, validate all constructed personae to ensure that their descriptions accurately describe the intended individuals. Validation should be carried out by seeking expert opinion on the correctness of the characteristics and goals described in each persona created, and on recommendations (if any) on how to improve the persona.

\section{Application of Proposed Method}

The proposed method was applied to construct a set of personae for designing a visual programming tool for children with ASC. Visual Programming lets users "specify a program in a two (or more) dimensional fashion" (Myers, 1990). It visualizes the process of program creation as well as program execution (Shu, 1988). Scratch is a visual programming tool created specifically for children aged between 8 - 16 to learn to programme through creating meaningful projects such as games and animations (Maloney et al., 2010). It allows users to create projects using its inbuilt or imported sprites and sounds, and a collection of blocks representing programming instructions that can be put together to create programming scripts.

The use of Scratch and other visual programming tools created for children has been reported to have educational benefits (Kafai and Burke, 2015). However, children with ASC are missing from these reports. Furthermore, available visual programming tools may not be usable by children with ASC (Zubair et al., 2018)

In a step towards addressing this issue, the proposed personae creation method was applied to construct a set of personae for children with ASC for the purpose of designing an accessible and usable visual programming tool. The constructed personae will serve as user representatives, design targets, evaluation tools, and decision guidance tools during the design and development of the visual programming tool. The data collection procedure, personae creation tasks and emerging results are summarised below:

\subsection{Collection of Data}

After conducting a literature search, six existing autistic personae (Leal et al., 2016; Mathisis, 2016) were chosen for use as the existing personae set. Only descriptive personae were selected, personae that consisted of lists of attributes or characteristics were dismissed.

Observational data of five (5) children with ASC collected during an initial study on evaluating the usability of Scratch for children with cognitive impairments (Zubair et al., 2018) was used for this study. The data consists of video screen recordings of each participant using Scratch to create stories.

Data were also collected through interviewing seven (7) experts that include two teachers and five academic researchers. The two teachers have more than eleven years of special education teaching experience between them. The five researchers included a professor (research interests in technology and disability), three associate professors (research interests in special education needs and disabilities) and a senior researcher (research interests in technology and autism). Each expert was interviewed separately, and data from the interview was recorded for analysis. Once the personae were constructed all seven experts were sent the personae and a questionnaire for validation (see Task 8 below). At the time of writing four of the seven experts have responded with their feedback.

Ethical approval was sought and received from Nottingham Trent University's Ethics Committee before interviews and all other data collection activities were conducted. Steps were also taken to ensure anonymity and confidentiality of participants. 


\subsection{Application of Tasks}

Below is a summary of the application of the method's tasks and their results:

Tasks 1 \& 2: The observational data gathered from five users (U1, U2, U3, U4 and U5) was analysed to identify behavioural variables. In addition to Cooper's suggestions for behavioural variables types, two new types ("Difficulties" and "Problem-solving strategies") were introduced to accommodate all the behavioural variables identified. In total, twenty-five (25) behavioural variables were identified, and they were grouped under seven (7) behavioural variable types (see Table 1 for examples).

Table 1 showing examples of identified behavioural variables and their types.

A five-point Likert scale was used to map each user to each behavioural variable. The scale described each user as either being "almost always", "often", "sometimes", "seldom" or "never" engaged in each behaviour represented by a behavioural variable.

Task 3, 4, \& 5: These tasks required expert input, and the seven participating experts were interviewed separately for these tasks. During each interview, the interviewee was provided with the mapping of users to behavioural variables and a set of existing personae. They were also given a quick tour of Scratch, its features and functionalities.

Each expert was asked to examine the mapping of users to behavioural variables and group users that have similar patterns of behaviour. The expert was then asked about the characteristics and goals related to each significant behavioural pattern for each group of users. The characteristics and goals for each group were listed, and the expert was asked to find personae from the provided set of existing personae with similar characteristics and goals (if any exists).

Once all interviews were conducted, all resulting data was analysed. Three persona abstracts emerged from the results: persona abstract 1 representing $\mathrm{U} 1$ and $\mathrm{U} 2$, persona abstract 2 representing U3 and U4 and persona abstract 3 representing U5. Characteristics and goals were assigned to each persona abstract from the data (see Table 2 for example). Names and dates of births for the persona abstracts were assigned randomly. Persona abstract 1 was named Oliver, persona abstract 2 was named Lilly and persona abstract 3 was named Ralph. The results also showed that no single existing persona closely resembles any of the three persona abstracts, although specific characteristics from multiple existing personae can be put together to form each of the persona abstracts.

Table 2 showing some characteristics and goals of a persona abstract (Ralph).

Task 6: None of the personae in the set of existing personae was similar enough to an abstract persona to allow for extension. Since the criteria for extending an existing persona was not met, there was no need for this task.

Task 7: For each of the three persona abstracts created so far, the characteristics and goals listed in Task 4 were expanded through descriptions to form full personae. Descriptions were provided in a third person format, starting with a short background story before describing the listed goals and characteristics.

Task 8: The three constructed personae together with a questionnaire were sent out to each of the experts that were interviewed. The descriptions in each persona were broken down into five sections to ease validation. Experts were asked to provide their level of agreement for the descriptions in each section using a four-point Likert scale. They were also asked for 
recommendations (if any), that could improve the overall quality of the personae as documents describing children with ASC. At the time of writing four (4) out seven (7) responses have been received, and there is universal agreement on the descriptions of 'Oliver' and 'Ralph', but 'Lilly' has two sections that caused disagreements in two experts. The received responses also included an interesting amount of qualitative feedback on how to improve the quality of all three personae. The feedback included suggesting additional content (e.g. goals) and modifying content (e.g. rewording descriptions) to convey a more accurate message.

\section{Analysis of the Proposed Method}

Based on the application of the proposed method and the results so far (described above), lessons were learnt, observations and reflections were made that can be used to inform improvements on both the method and how it should be applied. These are summarised below:

\subsection{Required Data, Sources and Collection}

The results collected so far have further confirmed the efficiency of data grounded persona construction methods. Upon reflection, it is clear how instrumental experts were in uncovering characteristics and goals associated with the observed actions and behaviours of users. Without the help of experts, the same value could not have been extracted from the user data. However, one modification that could be used in better-utilising experts in this process is to have focus groups rather than individual interviews. This could potentially lead to participants reacting to each other's beliefs and opinions and subsequently lead to more interconnected data (Kitzinger, 1994).

It was also observed that the data collected and used can be categorised based on its purpose in the persona creation process, this led to the introduction of the terms Base Data, Supplementary Data, Fictional Data and Validation Data. Base data is the data collected from users for the purpose of identifying behaviours and generating user mappings to behaviours, it provides the basic information that the persona is built from. Supplementary data is data from experts that either adds to base data or clarifies and interprets it. Fictional data consists of the imaginary personal details added to a persona to make it seem human and relatable. Finally, validation data is data collected from experts to validate and improve created personae.

\subsection{Extending Existing Personae}

The method proposed in this work encourages the creation of real-data based personae. It recommends the use of observational user data and seeking experts to provide and analyse data. However, it was observed that an unnecessary risk of contaminating real user characteristics and goals gathered from data, was taken in the attempt to extend existing personae. At the end of Task 4, persona abstracts contained a list of user characteristics and goals purely based on user data and expert knowledge. Extending a similar existing persona in Task 5 to fully describe a persona abstract could produce a final persona with inherited characteristics and goals that may not be true for the users represented by the persona abstract. A number of reasons can cause this such as extending a persona not built on real data or extending a persona that represents a larger group of users than the persona abstract.

It should be noted though, that this does not mean existing personae are not entirely useless in this creation process. They can be used as foundations to build new personae in situations where enough qualitative data is not available. For example where sufficient data collection activity is not feasible due to limited time, resources, or access to users. They can also be used as verification tools to verify and validate observations made and the results of data analysis. 
However, care must be taken in choosing the existing personae used for such purposes. They must be based on a sound methodical approach to analysing real user data. Otherwise, their inclusion could risk the validity of the end product.

\subsection{Validation and Feedback}

In the final stage of the method application described in section 3.2, feedback was sought from experts on the correctness of the descriptions contained in the three personae constructed, and recommendations (if any) for improving them. Although at the time of writing, not all experts have responded, an interesting amount of qualitative feedback and recommendations have been received on improving the quality of the personae. Once all responses are gathered, time will be required to carefully analyse, prioritise and implement these recommendations. Therefore, an additional step specifically for implementing feedback could prove useful in stressing the importance of utilising the feedback received from experts.

\section{Revised Method}

Taking the observations and suggestions presented above into account, a revised version of the persona creation method is proposed. It should be noted that these are major revisions that include the elimination of two tasks and the introduction of a new one. The tasks eliminated are the tasks associated with comparing and extending existing personae (Task 5 and Task 6). The new task introduced is for implementing feedback gathered during the process of validating personae with experts. Further validation can be conducted after implementing the feedback if required. The complete method is illustrated in Figure 2.

Figure 2 showing the revised personae creation method based on Cooper et al.'s (2007) method of constructing personae

Figure 2 is a visual representation of the revised method for creating personae for children with autism spectrum condition. It outlines the seven (7) tasks involved, the sequence in which they occur, and the "required data" for each task. Required data can be one or a combination of the following: base data, supplementary data, fictional data and validation data. These data types and how they can be sourced are briefly discussed below:

- Base Data: This data is collected before the persona construction process begins. It is collected directly from users through observations and forms the foundation for all personae to be constructed on.

- Supplementary Data: This data is collected during the persona construction process. It is collected from experts through discussions on user needs and goals. And, by getting their opinions on the results of analysing base data. Interviews or focus groups can be used to gather supplementary data.

- Fictional Data: Although the characteristics and goals of personae are extracted from real user data, fictional details such as personal information are needed to add life to personae and make them more relatable. Fictional data for building personae can be generated from user generation sites on the internet.

- Validation Data: Validation data is collected by gathering expert opinion on the constructed personae through interviews, focus groups, surveys, or questionnaires. Validation data not only rates the correctness of personae, but it also provides useful data that can lead to improvements in the quality of personae. Which is why it is very useful to collect qualitative feedback as part of validation data. 


\section{Conclusion}

This work proposed an approach to constructing personae for children with Autism Spectrum Condition (ASC). The method recommended the use of observational user data, data from experts, and existing personae to create and validate a new set of personae. After applying the proposed method to create personae for the design of visual programming tools, two major revisions were made to the method.

In making sure only real data is used in creating personae, the revision eliminated the possibility of extending an existing persona. This was done to avoid the risk of contaminating user characteristics and goals gathered from real data, with information from an existing persona that may not be built on real data or that represents a different user group. However existing personae can still be used as validation tools, as a source of data, or even as a foundation for a new persona in situations where real data is scarce. But care should be taken in choosing a persona with sound methodical and data origins.

The revised method also introduces an additional task for implementing the feedback gathered from experts during validation.

In summary, the revised method emphasizes the importance of user data as the primary source of information for constructing personae, and the value of engaging experts during the construction and validation process.

\section{Acknowledgement}

The authors are grateful to Nigeria's Petroleum Technology Development Fund for their financial support towards conducting this research.

\section{References}

Adlin, T. and Pruitt, J. (2010), The Essential Persona Lifecycle : Your Guide to Building and Using Personas, Morgan Kaufmann Publishers, San Francisco.

American Psychiatric Association. and American Psychiatric Association. DSM-5 Task Force. (n.d.). Diagnostic and Statistical Manual of Mental Disorders : DSM-5., available at: https://books.google.co.uk/books?hl=en\&lr=\&id=JivBAAAQBAJ\&oi=fnd\&pg=PT18\&dq=dsm+5\&ots=ceRP64MExa\&sig=EajsYwrROp86gbvHUaU_v k_lcBg\#v=onepage\&q=dsm 5\&f=false (accessed 25 December 2018).

Areej Al-Wabil, Sarah Drine, Sara Alkoblan, A.A. and Modhi Almozaini, R.A.-A. (2012), "The Use of Personas in the Design of an Arabic Auditory Training System for Children", Vol. 2 No. 2, p. 199.

Cooper, A. (1999), The Inmates Are Running the Asylum, Sams, Indianapolis, IN.

Cooper, A., Reimann, R. and Cronin, D. (2007), About Face 3: The Essentials of Interaction Design, Information Visualization, Vol. 3, John Wiley \& Sons, Indianapolis, IN.

Djajadiningrat, J.P., Gaver, W.W. and Fres, J.W. (2004), "Interaction relabelling and extreme characters", Proceedings of the Conference on Designing Interactive Systems Processes, Practices, Methods, and Techniques - DIS '00, ACM Press, New York, New York, USA, pp. 66-71.

Floyd, I.R., Cameron Jones, M. and Twidale, M.B. (2008), "Resolving Incommensurable Debates: a Preliminary Identification of Persona Kinds, Attributes, and Characteristics", Artifact, Vol. 2 No. 1, pp. 12-26.

Kafai, Y.B. and Burke, Q. (2015), “Constructionist Gaming: Understanding the Benefits of Making 
Games for Learning", Educational Psychologist, Vol. 50 No. 4, pp. 313-334.

Kitzinger, J. (1994), The Methodology of Focus Groups: The Importance of Interaction between Research Participants, Sociology of Health \& IIIness, Vol. 16, available at: https://onlinelibrary.wiley.com/doi/pdf/10.1111/1467-9566.ep11347023 (accessed 27 December 2018).

Leal, A., Teixeira, A. and Silva, S. (2016), "On the Creation of a Persona to Support the Development of Technologies for Children with Autism Spectrum Disorder", Lecture Notes in Computer Science (Including Subseries Lecture Notes in Artificial Intelligence and Lecture Notes in Bioinformatics), Vol. 9739, Springer, Cham, pp. 213-223.

Maloney, J., Resnick, M., Rusk, N., Silverman, B. and Eastmond, E. (2010), "The Scratch Programming Language and Environment", ACM Transactions on Computing Education, ACM, Vol. 10 No. 4, pp. 1-15.

McCrickard, D.S., Abel, T.D., Scarpa, A., Wang, Y. and Niu, S. (2015), "Collaborative design for young children with autism: Design tools and a user study", 2015 International Conference on Collaboration Technologies and Systems (CTS), pp. 175-182.

Myers, B. (1990), "Taxonomies of Visual Programming and Program Visualization", Visual Languages and Computing, Vol. 1 No. 1, pp. 97-123.

Norman, D. (2006), "Ad hoc personas \& empathetic focus", The Persona Lifecycle: Keeping People in Mind during Product Design, Morgan Kaufmann Press, pp. 154-157.

Shu, N.C. (1988), Visual Programming, Van Nostrand Reinhold, available at: https://dl.acm.org/citation.cfm?id=59606 (accessed 14 November 2017).

Vieira, D., Leal, A., Almeida, N., Silva, S. and Teixeira, A. (2017), “'Tell your day': Developing multimodal interaction applications for children with ASD", Lecture Notes in Computer Science (Including Subseries Lecture Notes in Artificial Intelligence and Lecture Notes in Bioinformatics), Vol. 10277 LNCS, Springer, Cham, pp. 525-544.

Zubair, M.S., Brown, D., Hughes-Roberts, T. and Bates, M. (2018), "Evaluating the Accessibility of Scratch for Children with Cognitive Impairments", Lecture Notes in Computer Science (Including Subseries Lecture Notes in Artificial Intelligence and Lecture Notes in Bioinformatics), Vol. 10907 LNCS, pp. 660-676. 


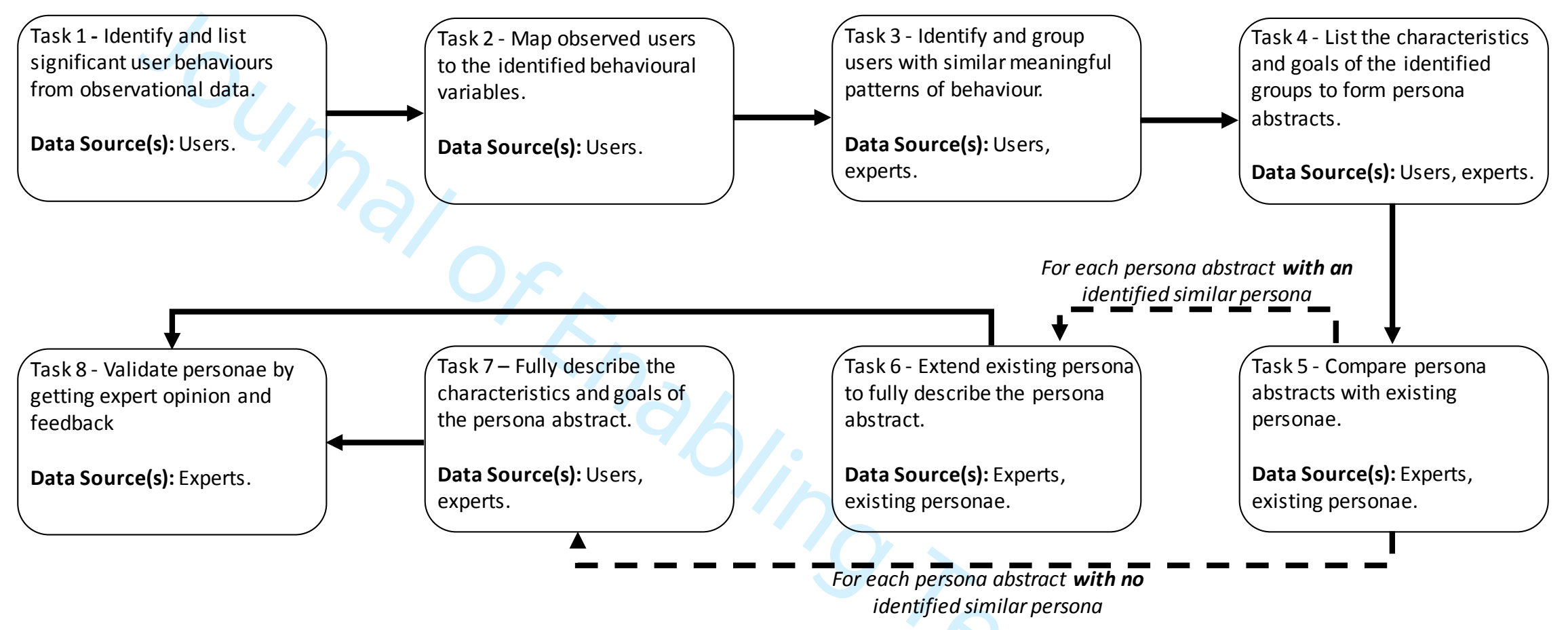

Figure 1 showing the proposed method based on Cooper et al.'s (2007) method of constructing personae. 


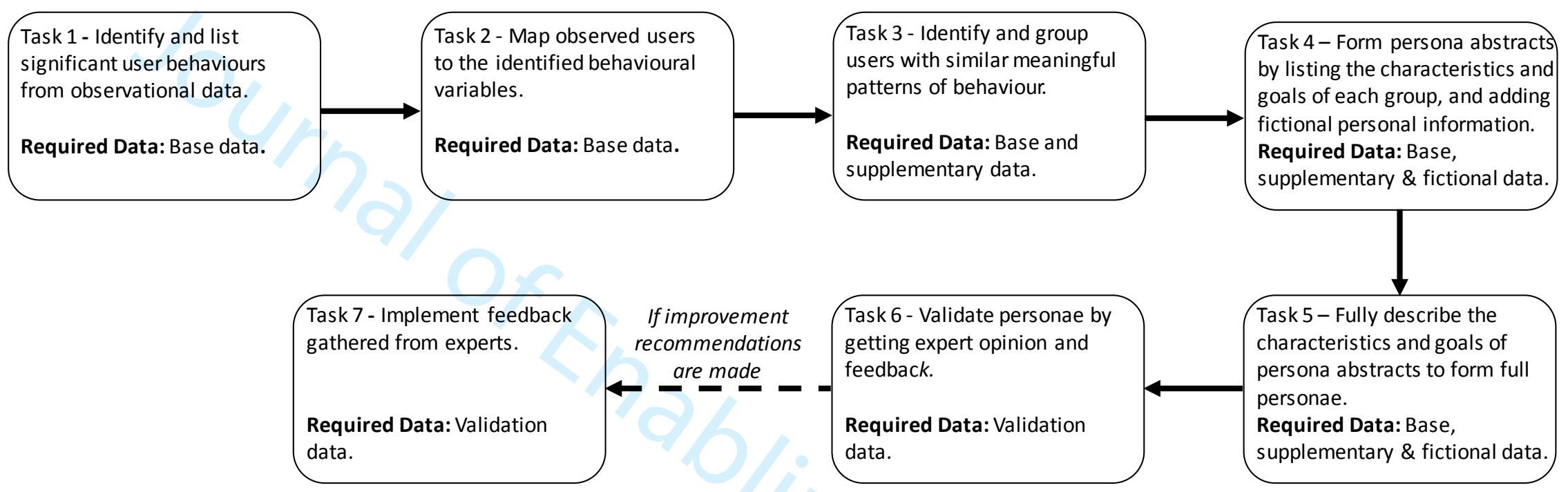

Figure 2 showing the revised personae creation method based on Cooper et al. 's (2007) method of constructing personae 


\begin{tabular}{|l|l|}
\hline Behavioural Variable Type & Behavioural Variable Example \\
\hline Activities & Adding sprites \\
\hline Aptitudes & Good reading and writing skills \\
\hline Motivations & Creating animations \\
\hline Skills & Comfortable operating a computer \\
\hline Attitudes & Likes interacting with the software \\
\hline Difficulties & Difficulty recognizing buttons \\
\hline Problem-solving strategies & Being idle \\
\hline
\end{tabular}

Table 1 showing examples of identified behavioural variables and their types. 


\begin{tabular}{|l|l|}
\hline Name & Ralph \\
\hline Date of birth & $13 / 06 / 2005$ \\
\hline Characteristics & - Has poor reading and reading comprehension skills. \\
& - Prefers visual presentation of information. \\
& - Has difficulty communicating needs. \\
& - Can comfortably operate computers and tablets. \\
& - Prefers communicating with symbols and pictures to communicating verbally. \\
& - Has restricted interests. \\
& - Has difficulty with the creative and imaginative aspects of projects. \\
& - Requires scaffolding from teacher/teaching assistant. \\
\hline Goals & - To be able to recognise buttons on the software. \\
& - To be able to work on projects more independently. \\
\hline
\end{tabular}

Table 2 showing some characteristics and goals of a persona (Ralph). 